MATEC Web of Conferences 44, 01035 (2016)

DOI: $10.1051 /$ matecconf/20164401035

(C) Owned by the authors, published by EDP Sciences, 2016

\title{
Design of Self-tracking High-pass Filter
}

\author{
Wei Zhao Zhang ${ }^{1,2, a}$, Jin Long $\mathrm{Li}^{3}$, Man Hong Fan ${ }^{1,2}$ and Sheng Qian Ma ${ }^{1,2}$ \\ ${ }^{1}$ College of Physics and Electronic Engineering, Northwest Normal University, 730070 Lanzhou, China \\ ${ }^{2}$ Key Laboratory of Atomic and Molecular Physics \& Functional Materials of Gansu Province, 730070 Lanzhou, China \\ ${ }^{3}$ College of Electronic Engineering, Lanzhou Institute of Technology, 730050 Lanzhou, China
}

\begin{abstract}
A novel self-tracking high-pass filter by using analog multiplier and current feedback op-Amp (CFA) is proposed in this paper. The frequency-voltage $(\mathrm{F} / \mathrm{V})$ converter transforms the input pulse signal into the voltage, and then the output of F/V converter controls the cut-off frequency of voltage controlled high-pass filter. Thus, the cut-off frequency of designed filter is linear with the frequency of input signal in case that resistors and capacitors are reasonable adjusted. The experiment and simulation results show that the cut-off frequency of designed filter is 1 kHz-100 kHz.
\end{abstract}

\section{Introduction}

Filters are widely used in information processing, automatic control, communications, and other fields. In the process of signal transmission, the signal is usually contaminated by noise. Using filtering technology can extract the useful signal, while suppressing the noise. The fixed cut-off frequency filter can be used in the situation of narrow range of the signal frequency. The frequency of the input signal often changes with the time. Therefore, the fixed cut-off frequency of the filter is difficult to meet the requirements. A self-tracking high-pass filter is proposed in this paper, and its cut-off frequency follows the frequency of the input signal.

There are three ways to regulate the cut-off frequency of filter. Firstly, the cut-off frequency of filter can be adjusted by variable resistors and capacitors $[1,2]$. In these filters, the cut-off frequency can only take the limited discrete value. What's more, variable resistors and capacitors may be cause unpredictable effects on signal amplitude and phase. Secondly, filter can be realized by the current mode technique, the cut-off frequency can be adjusted by bias current [3-6]. In these circuits, floating capacitor is used, and X terminal of the CCII is connected with a capacitor, which is easy to cause the circuit selfoscillates. Thirdly, the monolithic active filter chip, such as MAX26X, MAX29X, MAX27X, can be used [7, 8]. Although the cut-off frequency of these chips can be programmed (generally less than $1 \mathrm{MHz}$ ), it is difficult to realize the continuous adjustment of the cut-off frequency. In this paper, a new self-tracking high-pass filter is proposed. It is based on the high performance of $\mathrm{F} / \mathrm{V}$ converter LM331, the analog multiplier MLT04 and CFA (AD8001). The cut-off frequency of filter can automatically follows the frequency variation of input signal.

\footnotetext{
a Corresponding author: zhangweizhao163@163.com
}

\section{The principle of self-tracking high- pass filter}

The principle of self-tracking high-pass filter is shown in figure 1, which is composed of six parts: signal conditioning circuit, shaping circuit, F/V converter, programmable gain amplifier, control circuits based on FPGA and voltage controlled high-pass filter. At first, the input signal $\left(V_{i n}\right)$ amplified by signal conditioning circuit is converted into rectangular wave by shaping circuit. Since the bandwidth of $\mathrm{F} / \mathrm{V}$ converter is no more than $3 \mathrm{MHz}$, the frequency dividing circuit is designed, which scales down the frequency of input signal to the bandwidth range of $\mathrm{F} / \mathrm{V}$ converter. Then the output voltage of $\mathrm{F} / \mathrm{V}$ converter $\left(V_{f}\right)$ multiplied frequency dividing coefficients is frequency-voltage conversion of the input signal. At last, the cut-off frequency of highpass filter is continuously controlled by adjusting $V_{f}$. In order to realize the fast and real-time measurement, the frequency measurement circuit and frequency dividing circuit are designed by FPGA.

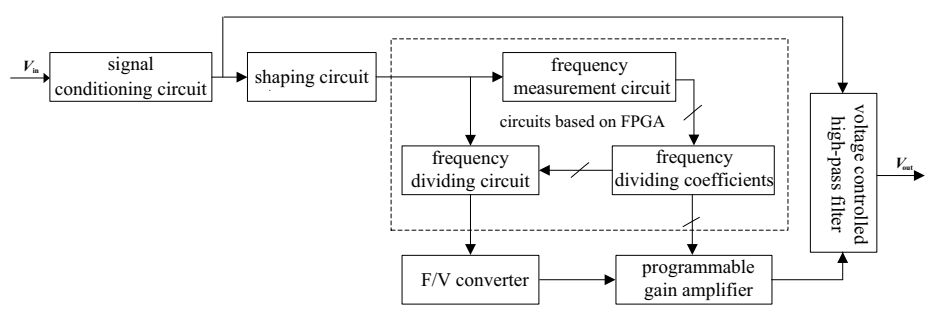

Figure 1. The principle diagram of self-tracking high-pass filter

\subsection{Wide-band signal conditioning circuit}

The wide-band signal conditioning circuit mainly realizes the gain adjustment of the input signal, including the 
variable gain control circuit and the fixed gain control circuit. The circuit diagram is shown in figure 2(a) and figure 2(b). The AD603 is a low noise, voltage-controlled amplifier for using in RF and IF AGC systems. It provides accurate, pin-selectable gains of $-11 \mathrm{~dB}$ to +31 $\mathrm{dB}$ with a bandwidth of $90 \mathrm{MHz}$ or $0 \sim+40 \mathrm{~dB}$ with a bandwidth of $30 \mathrm{MHz}$ or $+9 \mathrm{~dB}$ to $51+\mathrm{dB}$ with a bandwidth of $9 \mathrm{MHz}$. The variable gain control circuit consists of two pieces of cascaded AD603 at the gain of $-11 \mathrm{~dB}$ to $+31 \mathrm{~dB}$ with a bandwidth of $90 \mathrm{MHz}$. Thus, the voltage gain of $-20 \mathrm{~dB} \sim 60 \mathrm{~dB}$ can be realized by adjusting the voltage of VREF1_0 and VREF2_0. The gain of the fixed gain control circuit is $12 \mathrm{db}$.

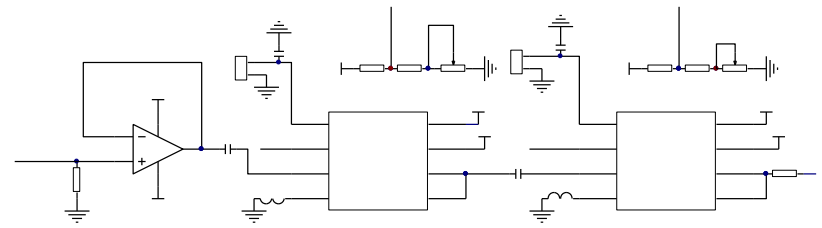

Figure 2(a). The variable gain control circuit

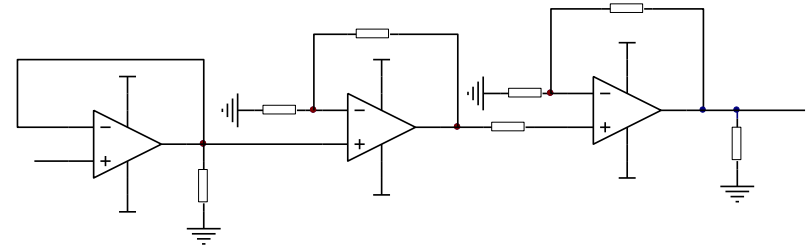

Figure 2(b). The fixed gain control circuit

The Amplitude-frequency characteristic curve of signal conditioning circuit measured by the 3577A HP network analyzer is shown in figure 3. The analysis shows that the $3 \mathrm{~dB}$ cut-off frequency of signal conditioning circuit can reach $70 \mathrm{MHz}$ with the $55 \mathrm{~dB}$ gain of voltage.

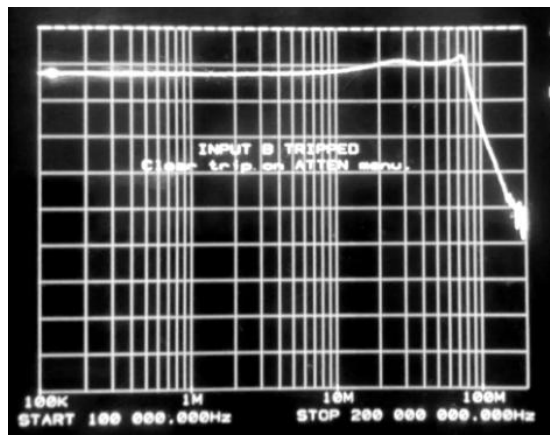

Figure 3. The Amplitude-frequency characteristic curve of signal conditioning circuit

\subsection{F/V converter}

$\mathrm{F} / \mathrm{V}$ converter transforms the input pulse signal into voltage output. The output voltage is linear with the frequency of input pulse signal. Thus, the frequency of input pulse signal can be measured indirectly by measuring the output voltage of $\mathrm{F} / \mathrm{V}$ converter. The $\mathrm{F} / \mathrm{V}$ converter consists of LM331, a few resistors and capacitors [9].
The relationship between the output voltage $V_{f}$ and the input frequency $f_{i}$ of $\mathrm{F} / \mathrm{V}$ converter can be expressed as:

$$
V_{f}=\frac{2.09 * R_{L} * R_{t} * C_{t}}{R_{s}} * f_{i}
$$

Where $R_{s}=R_{s 1}+R_{s 2}$.From (1) the output voltage $V_{f}$ is linear with the input frequency $f_{i}$ in case that resistors and capacitors are reasonable adjusted.

\subsection{Analog multiplier}

The MLT04 is a complete, four-channel, voltage output analog multiplier. The input voltage range of MLT04 is $2.5 \mathrm{~V} \sim+2.5 \mathrm{~V}, \mathrm{DC}$ and $\mathrm{AC}$ is allowed. Without considering of the nonlinearity of MLT04, the output voltage of MLT04 can be given as:

$$
V_{w}=0.4 V_{x} * V_{y}
$$

The combination of MLT04 and operational amplifier can also constitute analog divider as shown in figure 4 :

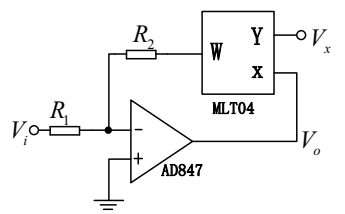

Figure 4. The analog divider

Defining equation can be given as:

$$
V_{0}=-\frac{2.5 R_{2} V_{i}}{R_{1} V_{x}}
$$

Here, $R_{1}=R_{2}$ is chosen, the equation can be written as:

$$
V_{o}=-\frac{2.5 V_{i}}{V_{x}}
$$

\section{The principle and design of self- tracking filter}

\subsection{The self-tracking first-order filter}

As shown in figure 5, the self-tracking first-order highpass filter circuit consists of $\mathrm{F} / \mathrm{V}$ converter, an analog divider, an operational amplifier, a few resistances and capacitances. U3 (MLT04), U4 (AD8001), a few capacitances and resistances constitute first-order voltage controlled high-pass filter. U1 (AD847) and U2 (MLT04) constitute divider. The control voltage $V_{f}$ is from the output of $\mathrm{F} / \mathrm{V}$ converter [10]. 


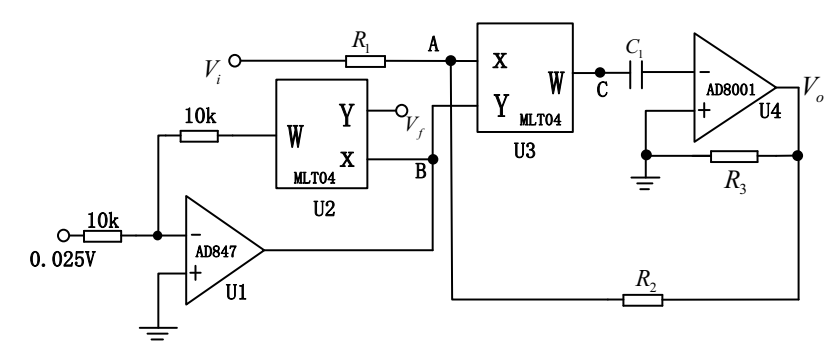

Figure 5. The self-tracking first-order high-pass filter

According to Kirchhoff's law and figure 5, the transfer function can be expressed as:

$$
H(s)=\frac{V_{o}}{V_{i}}=\frac{\frac{R_{2}}{R_{1}} s}{s+\frac{40 V_{f}\left(R_{1}+R_{2}\right)}{R_{1} R_{3} C_{1}}}
$$

Therefore, the $3 \mathrm{~dB}$ cut-off frequency $f_{0}$ and the gain factor $a_{0}$ of this transfer function can be given as:

$$
\begin{aligned}
& f_{0}=\frac{20 V_{f}\left(R_{1}+R_{2}\right)}{\pi R_{1} R_{3} C_{1}} \\
& a_{0}=\frac{R_{2}}{R_{1}}
\end{aligned}
$$

The sensitivity of components is $S_{R_{1}}^{f_{0}}=0, \quad S_{R_{2}}^{f_{0}}=1$, $S_{R_{3}}^{f_{0}}=S_{C_{1}}^{f_{0}}=-1$. It shows that the change of component value has little effect on the performance of filter. Here, $R_{1}=R_{2}=R_{3}=R$ is chosen, the equation (6) can be written as:

$$
f_{0}=\frac{40 V_{f}}{\pi R C_{1}}
$$

The equation (9) can be derived from equation (8) and equation (1):

$$
f_{0}=\frac{40 * 2.09 R_{t} R_{L} C_{t} f_{i}}{\pi R C_{1} R_{s}}
$$

From (9), it can be known that the $3 \mathrm{~dB}$ cut-off frequency $f_{0}$ is linear with the input frequency $f_{i}$ in case that resistors and capacitors are reasonable adjusted.

\subsection{The self-tracking second-order filter}

As shown in figure 6, a second-order voltage controlled high-pass filter is implemented by using three AD8001s, two MLT04s, a few resistances and capacitances. The control voltage $V_{f}$ is from the output of $\mathrm{F} / \mathrm{V}$ converter.

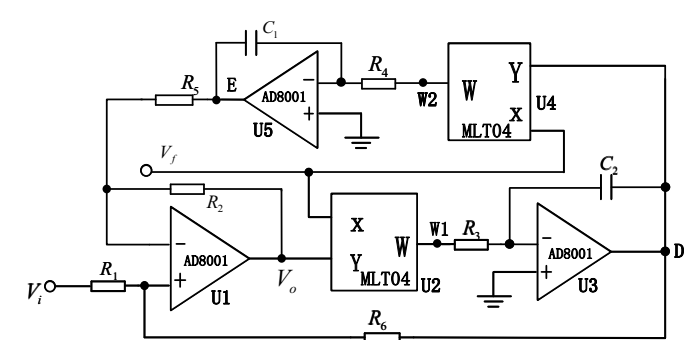

Figure 6. The self-tracking second-order high-pass filter

According to Kirchhoff's law and figure 6, the transfer function can be expressed as:

$$
H(S)=\frac{\frac{R_{6}\left(R_{2}+R_{5}\right)}{R_{5}\left(R_{1}+R_{6}\right)} s^{2}}{s^{2}+\frac{V_{f} R_{1}\left(R_{2}+R_{5}\right) s}{2.5 R_{3} R_{5}\left(R_{1}+R_{6}\right) C_{2}}+\frac{V_{f}^{2} R_{2}}{(2.5)^{2} R_{3} R_{4} R_{5} C_{1} C_{2}}}
$$

Therefore, the $3 \mathrm{~dB}$ cut-off frequency $f_{0}$ and the gain factor $a_{0}$ of this transfer functions can be given as:

$$
\begin{aligned}
& f_{0}=\frac{V_{f}}{5 \pi} \sqrt{\frac{R_{2}}{R_{3} R_{4} R_{5} C_{1} C_{2}}} \\
& a=\frac{R_{6}\left(R_{2}+R_{5}\right)}{R_{5}\left(R_{1}+R_{6}\right)}
\end{aligned}
$$

The sensitivity of components is $S_{R_{2}}^{f_{0}}=\frac{1}{2}$, $S_{R_{3}}^{f_{0}}=S_{R_{4}}^{f_{0}}=S_{R_{5}}^{f_{0}}=S_{C_{1}}^{f_{0}}=S_{C_{2}}^{f_{0}}=-\frac{1}{2}$. It shows that the change of component value has little effect on the performance of filter. Here, $R_{2}=R_{3}=R_{4}=R_{5}=R$, $C_{1}=C_{2}=C$ is chosen, the equation (11) can be written as:

$$
f_{0}=\frac{V_{f}}{5 \pi R C}
$$

The equation (14) can be derived from equation (13) and equation (1):

$$
f_{0}=\frac{2.09 R_{t} R_{L} C_{t} f_{i}}{5 \pi R C R_{s}}
$$

From (14), it can be known that the $3 \mathrm{~dB}$ cut-off frequency $f_{0}$ is linear with the input frequency $f_{i}$ in case that resistors and capacitors are reasonable adjusted.

\section{Simulation result}

As the maximum input voltage of MLT04 is $2.5 \mathrm{~V}$, the $3 \mathrm{~dB}$ cut-off frequency of filter is $1 \mathrm{kHz}-100 \mathrm{kHz}$. As to first-order high-pass filter, when taking $C=C_{t}=10 \mathrm{nF}, R_{t}=910 \Omega, R_{L}=18.73 \mathrm{k} \Omega, R_{s}=14.25 \mathrm{k} \Omega$ and $R=31.84 k \Omega$, the equation (9) becomes to be $: f_{0}=f_{i}$.

When taking the frequency of input signal is $1 \mathrm{kHz}$, $5 \mathrm{kHz}, 10 \mathrm{kHz}, 50 \mathrm{kHz}, 100 \mathrm{kHz}$, the amplitude-frequency characteristic curve of first-order high-pass filter is shown in figure 7 by the PSPICE simulations. 


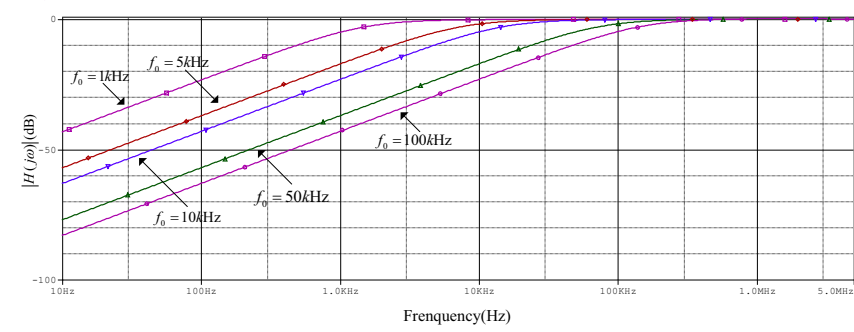

Figure 7. The amplitude-frequency characteristic curve of firstorder high-pass filter

When taking the frequency of input signal is $1 \mathrm{kHz}$, $5 \mathrm{kHz}, \quad 10 \mathrm{kHz}, \quad 50 \mathrm{kHz}, \quad 100 \mathrm{kHz}$, the experiments comparison of $f_{i}$ and $f_{0}$ of first-order high-pass filter is shown in table $1, \delta$ is the relative error.

Table1. The experiments comparison of $f_{i}$ and $f_{0}$ of firstorder high-pass filter

\begin{tabular}{c|c|c|c|c|c}
\hline$f_{i}(\mathrm{kHz})$ & 1 & 5 & 10 & 50 & 100 \\
\hline$f_{0}(\mathrm{kHz})$ & 0.991 & 4.898 & 9.887 & 49.885 & 99.882 \\
\hline$\delta(\%)$ & 0.9 & 2.04 & 1.13 & 0.23 & 0.12 \\
\hline
\end{tabular}

As to the second-order high-pass filter, when taking $C=C_{t}=10 \mathrm{nF}, R_{t}=910 \Omega, R_{L}=18.73 \mathrm{k} \Omega, R_{s}=14.25 \mathrm{k} \Omega$ and $R=159 \Omega$, the equation (14) becomes to be : $f_{0}=f_{i}$.

When taking the frequency of input signal is $1 \mathrm{kHz}$, $5 \mathrm{kHz}, 10 \mathrm{kHz}, 50 \mathrm{kHz}, 100 \mathrm{kHz}$, the amplitude-frequency characteristic curve of second-order high-pass filter is shown in figure 8 by the PSPICE simulations.

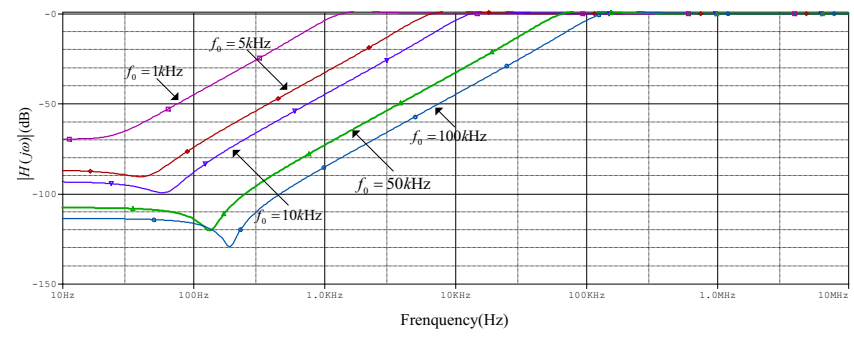

Figure 8. The amplitude-frequency characteristic curve of second-order high-pass filter

When taking the frequency of input signal is $1 \mathrm{kHz}$, $5 \mathrm{kHz}, \quad 10 \mathrm{kHz}, \quad 50 \mathrm{kHz}, 100 \mathrm{kHz}$, the experiments comparison of $f_{i}$ and $f_{0}$ of second-order high-pass filter is shown in table $2, \delta$ is the relative error.

Table2. The experiments comparison of $f_{i}$ and $f_{0}$ of secondorder high-pass filter

\begin{tabular}{c|c|c|c|c|c}
\hline$f_{i}(\mathrm{kHz})$ & 1 & 5 & 10 & 50 & 100 \\
\hline$f_{0}(\mathrm{kHz})$ & 0.989 & 4.892 & 9.881 & 49.878 & 99.872 \\
\hline$\delta(\%)$ & 1.10 & 2.16 & 1.19 & 0.24 & 0.13 \\
\hline
\end{tabular}

From the above simulation and experiment results, it can be seen that the cut-off frequency of designed filter can be adjusted automatically according to the input signal frequency.

\section{Conclusion}

In this paper, a self-tracking high-pass filter by using analog multiplier and CFA is proposed. Firstly, the F/V converter transforms the input pulse signal into output voltage $V_{f}$. Then the cut-off frequency of voltage controlled high-pass filter is controlled by $V_{f}$. The simulation and experiment results show that the cut-off frequency of designed filter is $1 \mathrm{kHz}-100 \mathrm{kHz}$. If using the higher frequency characteristics of analog multiplier (i.e., AD835), the cut-off frequency of filter can be extended $30 \mathrm{MHz}$.

\section{Acknowledgement}

The authors are thankful to the Natural Science Foundation of China (61162017) and Northwest Normal University NWNU-LKQN-13-16.

\section{References}

1. W. Z. Zhang, S. Q. Ma, X. P. Ran. Application of Electronic Technology, 37(07), 97-99 (2011).

2. J. Guo, Y. Jia, J. Teng, Q. Li. Chinese Journal of Scientific Instrument, 3(3), 295-297 (2006).

3. W. Sa-Ngiamvibool, B. Srisuchinwong. AEU International Journal of Electronics and Communications, 61(5), 307-313 (2007).

4. Z. Ali, T. Ali. International Journal of Electronics, 89(12), 913-923 (2002).

5. L. Y. Peng, Y. He, M. C. Huang, J. WU. Acta Electronica Sinica,31(8), 1234-1236 (2003).

6. A. M. Soliman. International Journal of Circuit Theory \& Applications, 35(4), 463-467 (2007).

7. S. M. Yin, B. Ji, H. L. Bao. J. L. Liu, J. G. Zhang. Application of Electronic Technology, 1(1), 54-56 (2012).

8. W. S. Wang. University of Electronic Science and Technology of China. (2010).

9. S. Q. Ma, X. P. Ran, M. H. Fan, W. Z. Zhang. Piezoelectrics \& Acoustooptics, 46(10), 969-971 (2013).

10. X. P. Ran, S. Q. Ma, J. F. Liu, W. Z. Zhang. Unifying Electrical Engineering and Electronics Engineering. Springer New York 4,1733-1741 (2014). 